\title{
Label-Free Immunochemistry Approach to Detect and Identity Antibiotics in Milk
}

\author{
FRANK DAVIS AND SÉAMUS P. J. HIGSON \\ Cranfield Health, Cranfield University, Bedford MK43 OAL, United Kingdom
}

\begin{abstract}
The rise of antibiotic-resistant bacteria is a major concern for the continued health and well-being of the general population. The widespread use of antibiotics within the farming industry is one of the factors that have been linked to the appearance of these resistant strains. Regulation exists to prevent antibiotic foodstuffs such as milk being distributed to consumers; however, for this to be effective, methods must exist for testing the milk to enforce these regulations. Current techniques are often time consuming and expensive, especially when applied to large number of samples. This has led to an interest in milk analyses, which can be carried out outside the laboratory. The development of immunosensors that exploit the exquisite specificity of antibody binding has been studied, both using labeled antibodies such as ELISA assays and, more recently, label-free approaches that directly detect the presence of the antibiotic on binding to a specific antibody. Within this review, we detail recent advances in the detection of antibiotics, especially in milk, using a variety of methods. Various techniques such as electrochemical and surface plasmon resonance methods are described.
\end{abstract} (Pediatr Res 67: 476-480, 2010)

$\mathrm{T}$ The use of antibiotic drugs is widely spread within our society, and these drugs have, over the years, saved many millions of lives. However, their use is not just restricted to the human population of the world, and they are widely used in veterinary medicine to treat a wide range of infectious diseases. However, concerns have been raised by the introduction of such compounds into the food chain, namely that it could lead to continual low dosing of the consumers of the animal products (meat, milk, etc) with antibiotics and may lead to the development of antibiotic resistant species of bacteria $(1,2)$. For example, fluoroquinolone drugs, such as ciprofloxacin, are broad-spectrum antibiotics active against many bacteria including anthrax. Many of these fluoroquinolones are added to farm animal feed because they can lead to greater and more rapid weight gain. The use of fluoroquinolones has been thought to have enabled the rise of resistant species of bacteria (3) in addition to a number of other problems such as chloramphenicol displaying bone marrow toxicity and penicillin causing a number of allergic reactions.

In a response to these concerns, maximum residue limits (MRL) have been set for many of these antibiotics in various foodstuffs. Milk is one of the most widely consumed food-

Received November 2, 2009; accepted January 12, 2010.

Correspondence: Séamus P. J. Higson, Ph.D., Cranfield Health, Cranfield University, Bedford MK43 0AL, United Kingdom; e-mail: s.p.j.higson@cranfield.ac.uk

Supported by the European Community Framework VI NMP2-CT-2003-505485, "ELISHA" contract. stuffs within the world, and if animals are being treated with antibiotics, these drugs or their residues will be present in the milk and meat of these animals. Table 1 lists many of the common antibiotics and their maximum permissible residue limits in milk within the European Union (4).

To enforce these regulations and to ensure that consumers are protected from accidental consumption of contaminated milk, it is necessary to have strategies in place for the regular testing of large numbers of milk samples. Traditional methods usually involve methods such as microbial inhibition assays or enzymatic colorimetric assays along with immunoassay methods such as ELISA (5). Usually, milk samples will be examined using rapid screening methods that may only indicate whether antibiotics are present and not the levels and types of antibiotics. Samples that fail to pass the screening tests will then be examined using more complex instrumental methods, such as HPLC and mass spectrometry, to identify and quantify which antibiotics are present. More recently, immunoassay type methods have been developed, which are faster and more specific than traditional screening methods. These ELISAs can identify and quantify a wide range of antibiotics and can be specific to a single or a group of antibiotics (6). Multi analyte ELISAs are available, which can simultaneously screen for a variety of antibiotics (6). However, ELISAs do require modification of the antibodies used in the assay and can become laborious and time consuming when large numbers of samples are to be analyzed.

Therefore, for this reason, there has been an interest in the development of sensors for antibiotics that do not require extensive chemical modification or pretreatment steps and that are rapid, quantitative, and selective. This has led to investigations into the use of immunosensors for the detection of pesticides. Most immunosensors consist of three principle components, as described later and detailed in Figure 1:

i. The first of these includes a receptor species, usually an antibody because of the high selectivity and specificity of antibody-antigen reactions, capable of recognizing and binding the analyte of interest with a high degree of selectivity.

ii. The second component that must be present is a transducer onto which the recognition element is immobilized enabling the translation of the binding event into a measur-

\footnotetext{
Abbreviations: AC, alternating current; MRL, maximum residue limits; SPR, surface plasmon resonance; WIOS, wavelength interrogated optical sensor
} 
Table 1. Maximum Residue Limits of Common Antibiotics in Milk Within the EU

\begin{tabular}{lc}
\hline \multicolumn{1}{c}{ Antibiotic } & Maximum residue limit $\left(\mathrm{ng} \cdot \mathrm{mL}^{-1}\right)$ in milk (EU 1994) \\
\hline Nermycin & 1500 \\
Gentomycin & 100 \\
Kanamycin & 150 \\
Streptomycin & 200 \\
Eurofloxacin & 100 \\
Ciprofloxacin & 100 \\
Chloramphenicol & 0 \\
Sulfamethazine & 100 \\
Penicillin G & 4 \\
Dihydrostreptomycin & 200 \\
Cephalexin & 100 \\
Tetracycline & 100
\end{tabular}

EU, European Union.

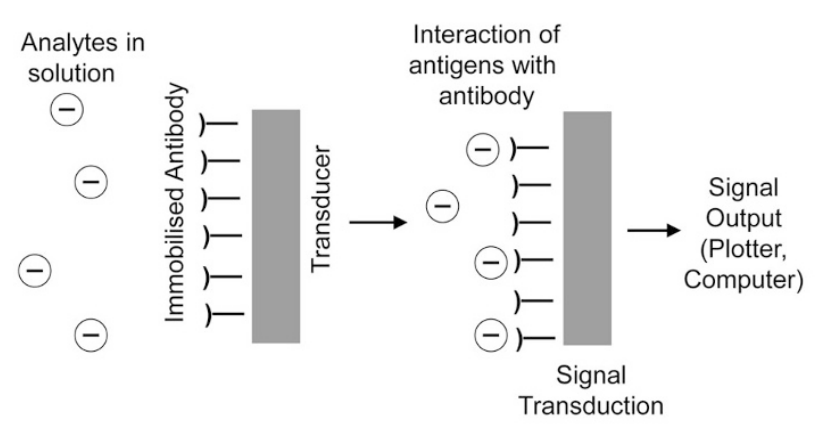

Figure 1. Schematic of an immunosensor.

able physical change; possible events include simple physical changes, such as a change in conductivity, refractive index, or simply film thickness, or mass changes.

iii. The third is that there must be inclusion of a method for measuring the change detected at the transducer and converting this into useful information. There are several methods; however, the two most commonly used are electrochemical and optical in nature.

There are two basic types of immunoassay: direct assays in which the antibody is bound to the transducer, and the binding of the antigen is measured. Moreover, there are competitive immunoassays in which an antigen, such as an antibiotic, is bound to the transducer. A known amount of the antibody is mixed with the sample under investigation. If no antigen is present, the antibody just binds to the antigen at the surface. However, if antigen is present in the sample, there is a competition between the free and immobilized antigens for the antibody, leading to an inhibition of surface antibody binding. Either technique combined with careful calibration is capable of accurate and specific determination of antigen concentrations.

\section{ELECTROCHEMICAL SENSING OF ANTIBIOTICS}

Electrochemical methods have been shown to be capable of detecting antibiotics because of their interaction with antibodies. For example, a recent publication (7) details the use of a horseradish peroxidase-based biosensor, which was capable of the oxidation of catechol. The presence of ciprofloxacin inhibited this reaction, and the extent of this inhibition could be used to determine the ciprofloxacin levels in buffer between
0.02 and $65 \mu \mathrm{M}$; however, other piperazine-based compounds could interfere with this assay.

Within our group, we have developed a direct label-free method for the electrochemical determination of ciprofloxacin. This used inexpensive screen-printed carbon electrodes as a base on which a film of conductive polyaniline was deposited. The polymer was then modified by a reaction with a commercial biotinylation agent, biotinamidohexanoicacid-3sulfo- $N$-hydroxysuccinimide, which reacted with free amine groups on the polymer to graft biotin groups onto the electrode surface. Exposure of this material to neutravidin led to the deposition of a layer of neutravidin onto the surface and held there by the strong avidin-biotin interaction. Meanwhile, antibodies that had been raised against ciprofloxacin were separately biotinylated and then immobilized from solution onto the composite material surface. This modified electrode was then investigated as an immunosensor for ciprofloxacin (8).

The electrodes were placed in phosphate buffer and interrogated by an alternating current (AC) impedance protocol between 1 and $10,000 \mathrm{~Hz}( \pm 5 \mathrm{mV}$ amplitude perturbation) offset to the formal potential of the redox probe $(+0.12 \mathrm{~V}$ versus $\mathrm{Ag} / \mathrm{AgCl}$ ). A mixture of $5 \mathrm{mmol} \cdot \mathrm{L}^{-1}$ each of $\left[\mathrm{Fe}(\mathrm{CN})_{6}\right]^{3-14-}$ was used as a redox mediator. Baseline traces were in the phosphate buffer containing the mediator but no antigen, and they were subsequently exposed to a range of concentrations of ciprofloxacin in $\mathrm{pH} 7.4$ phosphate buffer containing $\left[\mathrm{Fe}(\mathrm{CN})_{6}\right]^{3-/ 4-}$. Because nonspecific binding of many species, especially in complex media, can be a major problem in immunoassays, similar electrodes containing nonspecific $\operatorname{IgG}$ antibodies were used as a control to negate the effect of nonspecific adsorptions.

We have demonstrated previously $(8,9)$ that the most reproducible results can be obtained by measuring the resistive (real) component of the impedance and that the largest changes occur at low $(1 \mathrm{~Hz})$ frequencies. By comparing the impedance of the electrodes after exposure to antigen to their impedance before exposure, the percentage change in impedance could be obtained. Exposure of the electrode to ciprofloxacin solutions in phosphate buffer caused a drop in impedance. Calibration plots could be constructed showing the change of the real part of the impedance at $1 \mathrm{~Hz}$ against the ciprofloxacin concentration. Subtraction of the $\mathrm{IgG}$ response from the specific response gave "corrected" calibration plots as shown in Figure 2A. As can be seen, there is a logarithmic relationship between the change in $\mathrm{AC}$ impedance and the concentration of the antibiotic (8) in the range 1 to 100 $\mathrm{ng} \cdot \mathrm{mL}^{-1}$; above this level, the responses reach a plateau and the sensor seems saturated.

These results were obtained in phosphate buffer; however, the same technique could be applied in spiked milk samples (9). One problem was that very large nonspecific binding was observed for the IgG control, and it was realized that with a mixed antibody and a complex media such as milk, some of the antibodies will specifically bind to certain species in milk. Control electrodes were instead made using a specific antibody to prostate-specific antigen and showed low binding. The corrected calibration plot is shown in Figure 2, and it again displays a logarithmic relationship between change in AC 
A

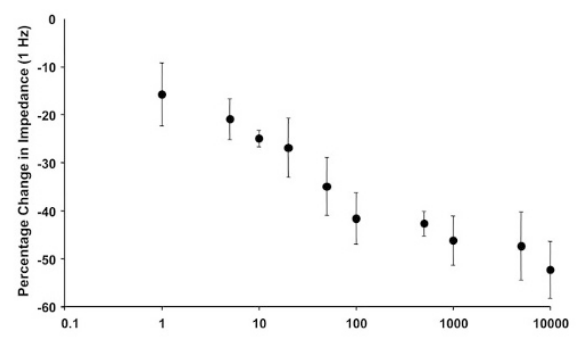

B

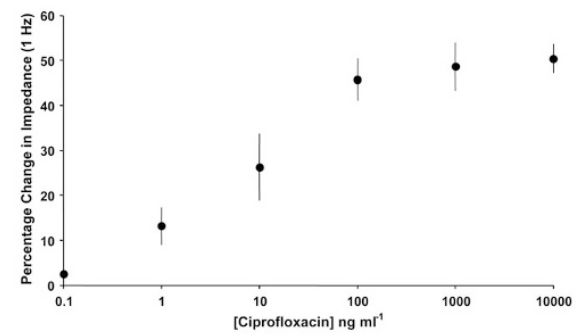

Figure 2. Corrected calibration curve for anti-ciprofloxacin modified electrodes in phosphate buffer $(A)$ and in milk $(B)$.

impedance and ciprofloxacin concentration in the range 0.1 to $100 \mathrm{ng} \cdot \mathrm{mL}^{-1}$. We have already noted that there are decreases in impedance for the electrodes after exposure to the antibiotic solution in phosphate buffer. What is of interest is that the opposite effect occurs on exposure to ciprofloxacin in milk, an increase in AC impedance on binding is observed.

One potential explanation for this is that in milk, a different ciprofloxacin species is being adsorbed because fluoroquinolone antibiotics, including ciprofloxacin, readily form calcium complexes with two ciprofloxacin units complexed to a single calcium ion (10). Binding of ciprofloxacin in either form is occurring, with the structural differences between the free and complexed forms of the antigen leading to different effects on the AC impedance results, potentially allowing differentiation between complexed and free ciprofloxacin. This is of interest because complexed form of ciprofloxacin is inactive; in this context, for example, the US Food and Drug Administration recommend that ciprofloxacin is not taken along with dairy products, calcium-fortified juices, or calcium-containing antacids (11) because they may lower the adsorption of the drug.

Other groups have studied electrochemical detection of antibiotics but have not used this in an immunoassay format. For example, a recent article described the adsorption of amoxicillin at multiwalled carbon nanotube electrodes and its determination by $\mathrm{AC}$ impedance and adsorptive stripping voltammetry (12). In addition, Langmuir-Blodgett or physically adsorbed layers of phospholipids could be deposited on gold electrodes and their electrical behavior shown to vary on exposure to Rifaximin (13).

\section{OPTICAL SENSING OF ANTIBIOTICS}

A more widely studied field of label-free sensing of antibiotics has been the use of antibodies combined with surface plasmon resonance (SPR) techniques. SPR dominates the field of optical biosensors, and commercial SPR systems are widely available, the major systems provider at the time of writing this article being Biacore AB (Uppsala, Sweden) (14). The

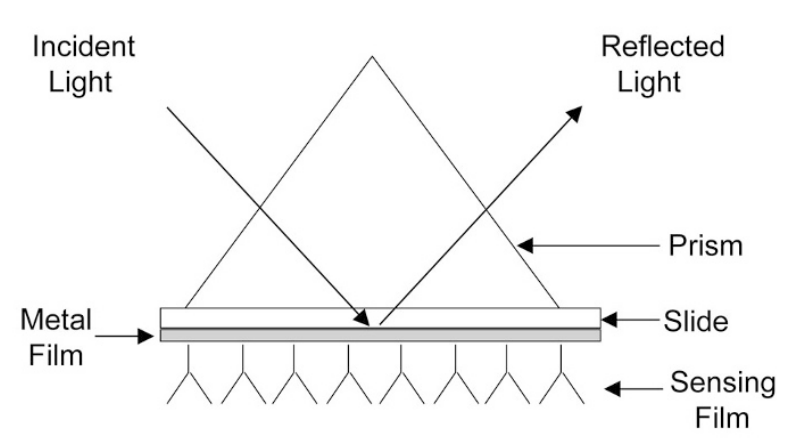

Figure 3. Schematic of a typical SPR apparatus.

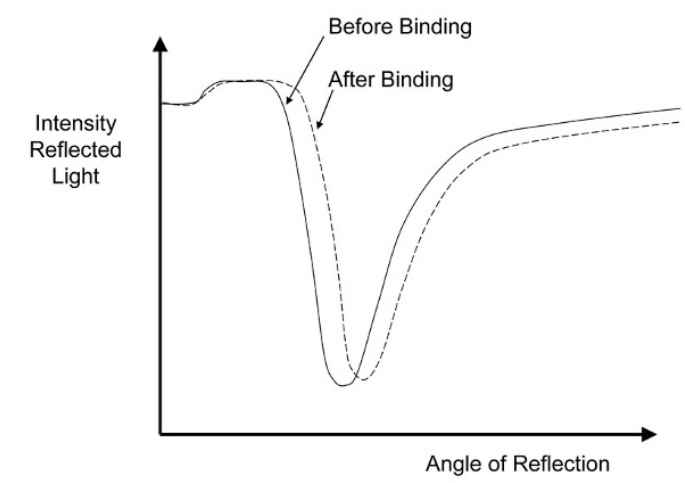

Figure 4. Shifts in the reflected light intensity curve with binding of analyte.

wide range of commercial instruments has made SPR a popular technique for studying intramolecular interactions. Several application areas are emerging and detailed reviews beyond the scope of this chapter have been published elsewhere $(14,15)$. SPR is a powerful technique to measure biomolecular interactions in real time by a combination of optical and electrochemical phenomena at metal surfaces and does not require the use of any labeling techniques. In SPR, a laser beam is focused onto the back of a gold film, (usually $\sim 40-\mathrm{nm}$ thick) deposited at the surface of a microscope slide clamped to a glass prism - with an index matching fluid placed between them or alternatively directly onto a gold-coated prism $(14,15)$. Figure 3 shows a schematic of a basic SPR set-up. At a critical angle of incidence, the laser energy is adsorbed to create surface plasmons, which are not strictly located at the metal surface but rather the intensity of their electromagnetic field decays exponentially into the adjacent medium. This phenomenon is known as the evanescent wave. At this angle, the reflected light intensity decreases dramatically, often to almost zero (Fig. 4). However, the position and the width of this adsorption are dependent on the evanescent wave and therefore are sensitive to the refractive index of the environment up to a distance of about a wavelength from the actual metal surface (15). Samples for SPR are usually in the form of a solution or dispersion. If recognition elements have been previously immobilized on the metal surface, then the binding of analyte molecules from the solution results in a change in the resultant SPR curve (Fig. 4). This change can, after suitable calibration, be used to determine the amount of antigen in the solution. 
In a previous study, an immunoassay for sulfamethazine was developed using SPR (16). A competitive immunoassay could be used to detect sulfamethazine; this antibiotic, for example, could be covalently immobilized at a gold surface that had been modified using carboxymethyl dextran (16). Exposing this chip to a sample of raw or skimmed milk containing a polyclonal antibody specific to the antibiotic along with sulfamethazine allowed determination of the sulfamethazine. Sensitivity was $<1 \mathrm{ppb}$, and the amount of the specific antibody required could be reduced by using a secondary antibody. Some cross reactivity with antibiotics of the same family was noted. Treatment of the chip with $\mathrm{NaOH}$ and $\mathrm{HCl}$ allowed regeneration of the surface. Aging the milk samples or the presence of bacteria was found to have only minimal effects of the results. Further study (17) showed by varying flow conditions and the antibody used, it proved possible to detect sulfamethazine in milk at levels as low as $1.7 \mathrm{ng} \cdot \mathrm{mL}^{-1}$.

A direct and also a competitive immunoassay for dihydrostreptomycin residues in milk were both developed (18). For the direct assay, a MAb was covalently bound to an SPR chip, whereas for the competitive assay, the antibiotic was conjugated with BSA and this conjugate immobilized at the chip surface in a similar manner. Again using a flow cell, both assays proved capable of detection of the antibiotic in milk at levels as low as 10 to $20 \mathrm{ng} \cdot \mathrm{mL}^{-1}$. Nonspecific binding of milk proteins proved a problem in this assay; however, ultrafiltration of samples reduced this problem and also the incorporation of a second channel, with no specific sensing layer, as a reference cancelled out many of these effects. Best results were obtained for a 5-fold dilution of milk in buffer and measured specific responses with minimal interference at $20 \mathrm{ng} \cdot \mathrm{mL}^{-1}$.

These initial studies only looked at single antibiotics; however, the development of a four-channel cell allowed the simultaneous investigation of gentamicin, neomycine, kanamycin, and a streptomycin derivative using a competitive assay (19). Each antibiotic was separately immobilized in a single channel and then a mixture of four specific antibodies, one for each compound in spiked milk samples was injected. The presence of any of the antibiotics in the sample was demonstrated by whether inhibition of antibody binding occurred. Limits of detection were 15 to $60 \mathrm{~nm} \cdot \mathrm{mL}^{-1}$ in 10 -fold diluted milk depending on the antibiotic. One advantage of this assay was that the chip could be washed by $0.2 \mathrm{M}$ $\mathrm{NaOH}$, which disrupted the antibody-antigen complex and regenerated the sensors. Total runtime including the regeneration step was only $7 \mathrm{~min}$ per sample and allowed simultaneous determination of all the antibiotics. It also proved possible to immobilize a conjugate of BSA with cephalexin, a $\beta$-lactam antibiotic, onto a SPR chip and use polyclonal antibodies to the antibiotic in a competitive assay (20). This system could be used for up to 40 detection/regeneration steps (using $0.01 \mathrm{M} \mathrm{NaOH}$ ) with only small losses in activity. Detection ranges of 4.88 to $2500 \mathrm{ng} \cdot \mathrm{mL}^{-1}$ and 244 to $3906 \mathrm{pg} \cdot \mathrm{mL}^{-1}$ were obtained in phosphate buffer and spiked milk samples, respectively.

As an alternative to the use of antibodies, an SPR-based biosensor was developed in which a well-characterized carboxypeptidase from Streptomyces R39 was used in an SPR assay (21). This enzyme hydrolyzes tripeptides to mono- and dipeptides, and this reaction is inhibited by penicillins. Samples containing tripeptides, the enzyme, and antibodies to dipeptides were used, and an SPR chip containing immobilized dipeptides was used. In this assay, the enzyme hydrolyzes the tripeptide to the dipeptide, which conjugates to the antibody in solution. If penicillin inhibits this reaction, the antibody conjugates to the surface-bound dipeptides only. The limit of detection of the assay for penicillin $\mathrm{G}$ was determined to be $2.6 \mathrm{ng} \cdot \mathrm{mL}^{-1}$ in milk, lower than the European Union MRL of $4 \mathrm{ng} \cdot \mathrm{mL}^{-1}$.

The use of lab-on-a-chip technology has also been investigated for the purpose of developing sensors for antibiotics in milk. Five types of antibodies, including those to penicillin G and dihydrostreptomycin, could be immobilized within a solgel film (22). This method was used to fabricate an array of sensing elements within a polydimethyl siloxane microfluidic device. This device was not strictly label-free, because a secondary antibody containing a fluorescent label was used, and the resultant antibody-antigen conjugates were being detected by fluorescence microscopy. Another lab-on-a-chip device has been recently reported (23), which combines the use of immobilized antibodies on a chip combined with a technique - wavelength interrogated optical sensing in which a collimated light beam is focused onto a dielectric waveguide on which is a grating coupler as shown in Figure 5. For a certain wavelength, a resonance occurs, generating an evanescent wave in the same manner as SPR. The output of this optical chip can also be measured in the same way that SPR can be. Sensing molecules such as antibodies are attached to
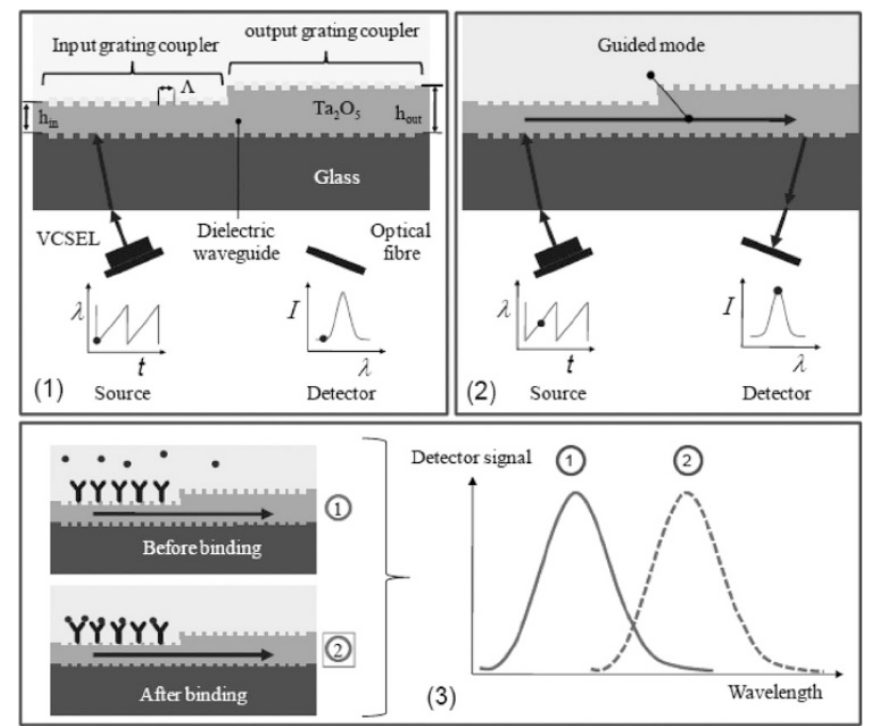

Figure 5. Sensing principles of WIOS technology based on grating waveguide resonant coupling. Characteristics of the WIOS system used are given by the following parameters: dielectric film thickness: $h_{\text {in }}(150 \mathrm{~nm})$ and $h_{\text {out }}(300 \mathrm{~nm})$; grating period: $L(360 \mathrm{~nm})$, grating groove depth $12 \mathrm{~nm}$. At resonance wavelength, a fraction of the incident light is transmitted into the waveguide and coupled out through the output pad. Output light is collected by plastic optical fibers and analyzed by a photodiode detector. Biofunctionalization of the sensing layer converts the WIOS into a biosensing device where the shift in the resonance wavelength reflects the quantity of bioanalyte bound. Reprinted from Suárez G et al. 2009 Lab Chip 9:1625-1630. Copyright (c) 2009 The Royal Society of Chemistry, with permission. 
the coupler, and on binding, it give rise to a change in refractive index, which affected the resonance wavelength. This set-up proved capable of the simultaneous detection of three antibiotic families - sulfonamides, fluoroquinolones, and tetracyclines-in raw milk at their MRL. The procedure could be automated and took $<10$ min per test. In another article, the wavelength interrogated optical sensor (WIOS) system was used to successfully test for a range of 30 antibiotics (24). In this study, the antibodies were synthesized to detect a class of antibiotics rather than single species. By using class-selective antibodies, the simultaneous screening for the most frequently used antibiotics in the veterinary field (e.g. sulfonamides, fluoroquinolones, $\beta$-lactams, and tetracyclines) was made possible in milk samples.

Other workers also used spotting technology to deposit various drugs as conjugates with ovalbumin at discrete locations on glass slides (25). These could then be used in a competitive assay where specific antibodies were bound to these conjugates. This technique again was not strictly labelfree as a fluorescent secondary antibody was then used, which allowed the system to be examined by fluorescence microscopy. This system proved capable of analyzing six samples each for eight antibiotics on a single chip within $3 \mathrm{~h}$ with accuracy and with sensitivities comparable with analyses using liquid chromatography combined with mass spectrometry assays.

In a development of SPR technology, the technique of SPR imaging - where the SPR behavior of a metal surface can be imaged-has been used to screen milk samples for seven different antibiotics simultaneously (26). Antibiotics were immobilized as spots onto commercial SPR chips. A competitive assay was used in which the chip was then exposed to antibodies to these materials. Levels of detection well below the MRL were established for most of these compounds in buffer and 10-fold diluted milk, although two of the antibiotics could not be detected below the MRL. In milk, there was some interference, probably because of nonspecific binding of milk proteins. The chip could be regenerated for multiple use by washing.

\section{CONCLUSIONS}

The detection of antibiotics in milk is a necessary procedure to help prevent the creation and spread of antibiotic resistance bacteria. Current methods such as chromatographic and mass spectral analysis require expensive equipment and highly trained staff. Other methods such as ELISAs are effective but unsuitable for large numbers of samples, because they are time consuming and require the use of labeled antibodies. Labelfree approaches offer many advantages, because they do not require the use of expensive labeling reagents and can often be carried out in the field. Both electrochemical and optical approaches show promise, being capable of detection of antibiotics at levels below the MRL. Both techniques could be used in array format where they can simultaneously test samples for a range of antibiotics. Other advantages include the small sample volumes required for these assays and no necessity for pretreatment of the samples before analysis. Electrochemical methods could use cheap screen-printed electrodes as throwaway single-shot sensors. Although optical chips are more expensive, their price would come down with large-scale manufacture, and in some cases reported in this study, it has been possible to regenerate the sensor for multiple usage. From this, it is expected that label-free approaches will become competitors to the current ELISA-based methods within the near future.

\section{REFERENCES}

1. Garrod LP 1964 Hazards of antibiotics in milk and other food products. Proc R Soc Med 57:1087-1088

2. Okolo MI 1986 Bacterial drug resistance in meat animals: a review. Int J Zoonoses 13:143-152

3. Gendrel D, Chalumeau M, Moulin F, Raymond J 2003 Fluoroquinolones in paediatrics: a risk for the patient or for the community. Lancet Infect Dis 3:537-546

4. Council of the European Communities 1990 Council regulation (EEC) no. 2377/90 laying down a Community procedure for the establishment of maximum residue limits of veterinary medicinal products in foodstuffs of animal origin. Official Journal of the European Communities L224:1-8. Available at: http://eur-lex.europa.eu/ LexUriServ/LexUriServ.do?uri=CELEX:31990R2377:EN:HTML, Accessed January $26,2010$.

5. Mitchell JM, Griffiths MW, McEwen SA, McNab WB, Yee AJ 1998 Antimicrobial drug residues in milk and meat: causes, concerns, prevalence, regulations, tests, and test performance. J Food Prot 61:742-756

6. Adrian J, Pinacho D, Granier B, Diserens DM, Sanchez-Baeza F, Marco MP 2008 A multianalyte ELISA for immunochemical screening of sulfonamide, fluoroquinolone and beta-lactam antibiotics in milk samples using class-selective bioreceptors. Anal Bioanal Chem 391:1703-1712

7. Torriero AA, Ruiz-Diaz JJ, Salinas E, Marchevsky EJ, Sanz MI, Raba J 2006 Enzymatic rotating biosensor for ciprofloxacin determination. Talanta 69:691-699

8. Garifallou GZ, Tsekenis G, Davis F, Millner PA, Pinacho DG, Sanchez-Baeza F, Marco MP, Gibson TD, Higson SP 2007 Labeless immunosensor assay for fluoroquinolone antibiotics based upon an AC impedance protocol. Anal Lett 40:1412-1422

9. Tsekenis G, Garifallou G-Z, Davis F, Millner PA, Pinacho DG, Sanchez-Baeza F, Marco MP, Gibson TD, Higson SP 2008 Detection of fluoroquinolone antibiotics in milk via a labeless immunoassay based upon an alternating current impedance protocol. Anal Chem 80:9233-9239

10. Upadhyay SK, Kumar P, Arora V 2006 Complexes of quinolone drugs norfloxacin and ciprofloxacin with alkaline earth metal perchlorates. J Struct Chem 47:1078-1083

11. CIPRO ${ }^{\circledR}$ (ciprofloxacin hydrochloride) tablets, CIPRO ${ }^{\circledR}$ (ciprofloxacin) Oral suspension. Available at: http://www.accessdata.fda.gov/drugsatfda_docs/label/2008/ 019537s067,020780s025lbl.pdf, Accessed January 4, 2010

12. Rezaei B, Damiri S 2009 Electrochemistry and adsorptive stripping voltammetric determination of amoxicillin on a multiwalled carbon nanotubes modified glassy carbon electrode. Electroanalysis 21:1577-1586

13. Morandi S, Puggelli M, Caminati G 2008 Antibiotic association with phospholipid nano-assemblies: a comparison between Langmuir-Blodgett films and supported lipid bilayers. Colloid Surf A Physicochem Eng Asp 321:125-130

14. Karlsson R 2004 SPR for molecular interaction analysis: a review of emerging application areas. J Mol Recognit 17:151-161

15. Ivarsson B, Malmqvist M 2002 Surface plasmon resonance development and use of BIACORE instruments for biomolecular interaction analysis. In: Gizeli E, Lowe CR (eds) Biomolecular Sensors. Taylor and Francis, Oxford, UK, pp 241-268

16. Sternesjo A, Mellgren C, Bjorck L 1995 Determination of sulfamethazine residues in milk by a surface plasmon resonance-based biosensor assay. Anal Biochem 226:175181

17. Gaudin V, Pavy ML 1999 Determination of sulfamethazine in milk by biosensor immunoassay. J AOAC Int 82:1316-1320

18. Haasnoot W, Loomans EE, Cazemier G, Dietrich R, Verheijen R, Bergwerff AA, Stephany RW 2002 Direct versus competitive biosensor immunoassays for the detection of (dihydro)streptomycin residues in milk. Food Agric Immunol 14:15-27

19. Haasnoot W, Cazemier G, Koets M, van Amerongen 2003 A single biosensor immunoassay for the detection of five aminoglycosides in reconstituted skimmed milk. Anal Chim Acta 488:53-60

20. Dillon PP, Daly SJ, Browne JG, Manning BM, Loomans E, van Amerongen A, O'Kennedy R 2003 Application of an immunosensor for the detection of the beta-lactam antibiotic, cephalexin. Food Agric Immunol 15:225-234

21. Gustavsson E, Bjurling P, Sternesjo A 2002 Biosensor analysis of penicillin G in milk based on the inhibition of carboxypeptidase activity. Anal Chim Acta 468:153-159

22. Choi JW, Kim YK, Kim HJ, Lee W, Seong GH 2006 Lab-on-a-chip for monitoring the quality of raw milk. J Microbiol Biotechnol 16:1229-1235

23. Suárez G, Jin YH, Auerswald J, Berchtold S, Knapp HF, Diserens JM, Leterrier Y, Månson JA, Voirin G 2009 Lab-on-a-chip for multiplexed biosensing of residual antibiotics in milk. Lab Chip 9:1625-1630

24. Adrian J, Pasche S, Pinacho DG, Font H, Diserens JM, Sanchez-Baeza F, Granier B, Voirin G, Marco MP 2009 Wavelength-interrogated optical biosensor for multianalyte screening of sulfonamide, fluoroquinolone, $\beta$-lactam and tetracycline antibiotics in milk. Trends Anal Chem 28:769-777

25. Chen A, Wang G, Cao Q, Wang Y, Zhang Z, Sun Y, Wang H, Xu C, Zhou Q, Han P, Liu M, Yang Y, Xing W, Mitchelson KR, Cheng J 2009 Development of an antibody hapten-chip system for detecting the residues of multiple antibiotic drugs. J Forensic Sci 54:953-960

26. Rebe Raz S, Bremer MG, Haasnoot W, Norde W 2009 Label-free and multiplex detection of antibiotic residues in milk using imaging surface plasmon resonancebased immunosensor. Anal Chem 81:7743-7749 